\title{
Capacidad de humectación de soluciones irrigantes del tratamiento de conductos radiculares. In vitro
}

Wettability of irrigating solutions for root canal treatment. In vitro

\section{Resumen}

Objetivo: Determinar y analizar la capacidad de humectancia de soluciones irrigantes usadas en el tratamiento de conductos radiculares sobre la superficie dentinaria de dientes de bovino. Materiales y método: Estudio experimental in vitro. Se preparó 60 bloques de acrílicoautocurado de $40 \mathrm{~mm} \mathrm{x}$ $30 \mathrm{~mm}$ x $8 \mathrm{~mm}$ donde una superficie expone tejido dentinario de diente de bovino. Los irrigantes de prueba fueron: solución de hipoclorito de sodio al $5,25 \%$, gluconato de clorhexidina al $0.12 \%$, suero fisiologico y agua de cal. En la superficie de dentina se depositó una gota calibrada de las soluciones irrigantes, estas gotas sésiles en contacto con dentina mostraron muchas formas las cuales fueron fotografiadas para posteriormente superponerle el trazo de una tangente desde el punto de contacto y sobre el ecuador del arco establecido, el ángulo resultante fue medido con un transportador estableciendo el ángulo de contacto. Resultados: Gluconato de Clorhexidina al 0,12\%, establecía un ángulo de contacto promedio de $41.33^{\circ}$, seguidos por el agua de cal: $43.23^{\circ}$, hipoclorito de sodio al $5,25 \%: 61.17^{\circ}$ y la solución salina al 9/1000 con $61.43^{\circ}$. Conclusiones: Se concluye que la clorhexidina y el agua de cal son los líquidos con mayor capacidad de humectancia sobre dentina de diente de bobino. Resultado no esperado pues por las características moleculares se creía que el hipoclorito de sodio tendría una mejor capacidad de humectancia.

Palabras clave: Humectancia; tensión Superficial; energía superficial; angulo de contacto; soluciones irrigadoras de conductos.

\begin{abstract}
Objective: the objective of this study was to determine and analyze the wettability of irrigating solutions of root canal treatment in bovine tooth dentin surface. Materials and method: For the study were prepared 15 acrylic blocks : $40 \mathrm{~mm}$ x $30 \mathrm{~mm}$ x $8 \mathrm{~mm}$ wherein a surface exposed tooth dentin of bovine tissue. There were used as test

irrigants: $5,25 \%$ sodium hypochlorite solution, $0,12 \%$ chlorhexidine gluconate, water saturated with calcium hydroxide and sodium chloride solution 9/1000. On dentin surface was deposited a calibrated drop of irrigating solution, this sessile drop contact

with dentin surface showed many forms which were photographed to measure them with a conveyor provided the angle (contact angle). Results showed that Chlorhexidine Gluconate 0,12\%, established a contact angle with an average of $41.33^{\circ}$, followed by water saturated with calcium hydroxide $\left(43.23^{\circ}\right)$, sodium hypochlorite $5,25 \%\left(61.17^{\circ}\right)$

and saline $9 / 1000$ with $61.43^{\circ}$.Conclusion: It is concluded that chlorhexidine and water saturated with calcium hydroxide are irrigants with better ability to humectancy on bovine dentin. Unexpected result because of the molecular characteristics it was believed to sodium hypochlorite have a better ability to humectancy.
\end{abstract}

Keywords: Wettability; surface tension; surface energy; contact angle; canal irrigating solutions.

\section{Introducción}

En los tratamientos endodónticos una etapa importante es la preparación quirúrgica de los conductos radiculares, está a su vez comprende la preparación biomecánica y químico-físico, cuyo objetivo es promover la disolución del tejido pulpar, el modelado y antisepsia de los sistemas de conductos radiculares, para conseguir éste propósito es necesario una acción asociada entre la instrumentación y el uso apropiado de las diferentes soluciones irrigadoras. Siempre ha sido tema de investigaciones las propiedades biológicas, químicas, antimicrobianas de las soluciones irrigadoras, los estudios han contribuido en conocer cuales tienen más propiedades sobre todo antimicrobianas, hasta ahora, la sustancia química ideal permanece desconocida ${ }^{1-3}$; sin embargo poco se conoce sobre su capacidad como irrigante, mejor dicho cuanto

\section{Artículo Original}

\section{Abel Anglas Machacuay ${ }^{1}$, Martha \\ Pineda-Mejía1, Doris Salcedo-Moncada ${ }^{1}$}

1. Departamento Académico Estomatología Rehabilitadora de la Facultad de Odontología de la Universidad Nacional Mayor de San Marcos, Perú.

Correspondencia:C.D. Abel Anglas Machacuay Correo electrónico: abelanglasm@yahoo.es Facultad de Odontología de la Universidad Nacional Mayor de San Marcos.

Av. Germán Amézaga 375, Lima 1, Perú

\section{Coautoras:}

Doris Salcedo- Moncada:

dsalcedom@unmsm.edu.pe

Martha Pineda-Mejía:

mpinedam@unmsm.edu.pe
Recibido: 10/03/15

Aceptado: 20/08/16 
bisbiguanida catiónica con propiedades antibacterianas, derivada de su atracción electrostática por la pared celular microbiana, lo que daña el equilibrio osmótico celular. La clorhexidina también tiene sustantividad debido a su capacidad para unirse en superficies cargadas negativamente en la cavidad oral, como el tejido dental mineralizante, la película adquirida, las membranas mucosas y los materiales restauradores, y liberarse lentamente ${ }^{4-8}$.

Maisto y Amadeo, citados por Lasala, recomiendan como irrigante una solución de saturación de hidróxido de cálcico en agua, la cual denominan lechada de cal o agua de cal, basados más en su efecto antibacteriano, debido a su alcalinidad que, en su capacidad proteolítica, que podría alternarse con el agua oxigenada, empleando como último irrigador la lechada de cal, lo cual, favorecería la reparación apical sobretodo en dientes con ápices abiertos ${ }^{9}$.

Solución salina: Es el irrigante más biocompatible que existe, en concentración isotónica, la solución salina no produce daños conocidos en el tejido y se ha demostrado que expele los detritos de los conductos, pero tiene poco o ningún efecto químico y depende solamente de su acción mecánica, para remover restos del conducto radicular. En general esta sustancia es la más suave con el tejido dentro las soluciones de irrigación. El efecto antibacteriano y su disolución de tejido es mínima si se compara con el peróxido de hidrógeno, o el hipoclorito de sodio.

Una solución irrigadora, empleada en alternancia con la instrumentación del conducto radicular debe estar en contacto íntimo con las paredes del conducto para promover la disolución y limpieza de restos de tejidos orgánicos la dentina y sus túbulos. Durante la instrumentación del conducto radicular, las áreas intactas son un problema común incluso con preparación rotatoria súper- elástica. Por ejemplo, los estudios en conductos mesiobucales de los primeros molares superiores refieren un promedio de áreas intactas entre 40,0\% y $47,4 \%{ }^{5-7}$. Por esta razón, la acción del irrigante y su actividad antimicrobiana en estas áreas son importantes para la correcta antisepsia del conducto radicular.

Cuando sólidos y líquidos entran en contacto, sus moléculas periféricas dan origen a fenómenos de superficie $e^{8-12}$. En este trabajo se estableció el comportamiento de las moléculas de una superficie solida (dentina de bovinos) denominada energía superficial y las moléculas periféricas de los líquidos empleados para la antisepsia y evacuación de restos de los conductos radiculares, denominado tensión superficial. Como fenómenos de superficie se pueden estudiar la capacidad de humectación o mojamiento, escurrimiento, penetrancia, capilaridad y otros.

Una solución química para el uso endodóntico debe mostrar una humedad sustancial, lo que aumenta su capacidad de disolvente y mejora la actividad antimicrobiana en áreas no instrumentadas del sistema de conductos radiculares ${ }^{8}$. La humectabilidad de un líquido puede evaluarse midiendo el ángulo de contacto del líquido con una superficie sólida y su tensión superficial ${ }^{12}$.

El presente estudio se realizó para evaluar la capacidad de humectación a través del ángulo de contacto de cuatro soluciones irrigantes del conducto radicular: $\mathrm{NaOCl}$ al 5.25\%, Gluconato de Clorhexidina al $0.12 \%$, agua de cal y suero fisiológico sobre dentina de dientes de bovino.

Según la bibliografía, los líquidos que humectan mejor son los de menor peso molecular y menor densidad, entonces hipotéticamente se planteó que el hipoclorito de sodio, tenía mejor capacidad de humectancia.

\section{Materiales y método}

La investigación efectuada evaluó la Capacidad de humectación de cuatro soluciones irrigantes de conductos radiculares (gluconato de clorhexidina, hipoclorito de sodio, agua de cal y solución salina o suero fisiológico) sobre dentina de dientes de bobino,

Se seleccionaron 60 piezas dentarias de bovinos, incisivos antero-superiores centrales y laterales las cuales una vez extraídos fueron mantenidos en condiciones ideales, hasta su procesamiento. Luego se procedió a cortar las piezas dentarias en el límite corono-radicular, las raíces se almacenaron en suero fisiológico hasta la confección de los especímenes de prueba para ángulos de contacto.( Fotografia 1)

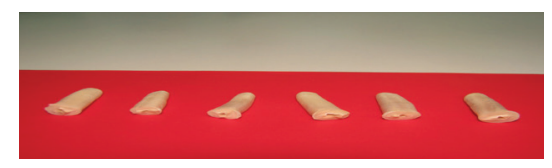

Fotografía 1: cortes de dientes de bobino

Posteriormente se confeccionaron 60 bloques de acrílico de autocurado de 40 $\mathrm{mm}$ x $30 \mathrm{~mm}$ x $10 \mathrm{~mm}$ en los cuales mientras polimerizaba se incorporaron las piezas radiculares de dientes de bovino, luego esta superficie fue desgastada quedando los bloques de acrílico en 40 $\mathrm{mm} \times 30 \mathrm{~mm} \times 8 \mathrm{~mm}$, todas las superficies paralelas, lisas y perpendiculares con sus correspondientes, quedando cada espécimen con una superficie dentinaria expuesta.

Para este experimento se probaron las siguientes soluciones irrigantes: $\mathrm{NaOCl}$ al 5,25\% (Hisol $5.25 \%$ ), gluconato de clorhexidina al $0.12 \%$ ( Perio Aid DENTAID), agua de cal (preparada ańadiendo medio gramo de hidróxido de calcio en polvo (Biodinámica-Brasil ) en 100 cc de agua destilada, después de la mezcla se dejó en reposo una hora antes de realizar la prueba) y suero fisiológico (Braum 1000 cc) (grupo control).

Durante el procedimiento se dejó una gota calibrada de las soluciones irrigantes (empleando un gotero de laboratorio) sobre un espécimen preparado con una superficie lisa de dentina, estas gotas sésiles en contacto con dentina mostraron muchas formas las cuales fueron fotografiadas para posteriormente, mediante un transportador, medir el ángulo de contacto que se establecía, entre la superficie del solido (dentina de bovino) y la tangente trazada desde el lugar de contacto de la gota con la dentina y que pasa por su ecuador, (grafico 1)

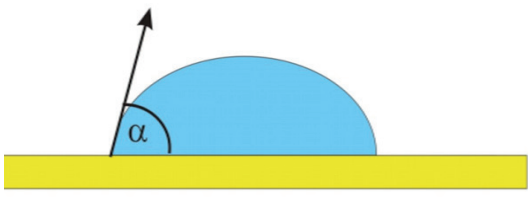

Grafico 1: alfa = Angulo de contacto

Se obtuvieron 60 fotografías, quince fotografías con cada solución irrigadora: La toma fotográfica se realizó inmediatamente dejado la solución irrigadora en la superficie dentinaria (hasta 10 segundos como máximo). La cámara fotográfica empleada fue NIKON D3100 con objetivo zoom $18-55 \mathrm{~mm}$. Todas las tomas fotográficas se realizaron en las mismas condiciones, Fig. 4,5,6,7.

Técnica de Recolección de Datos: las medidas de los ángulos de contacto se realizaron según el método de la gota sésil considerado el más adecuado para investigaciones. Con las fotografías obtenidas se procedió a presentar en power point, sobre cada fotografía se graficó una tangente desde el punto de contacto del líquido con la superficie dentinaria, por sobre el arco establecido por la gota, registrándose 60 valores de ángulos. 


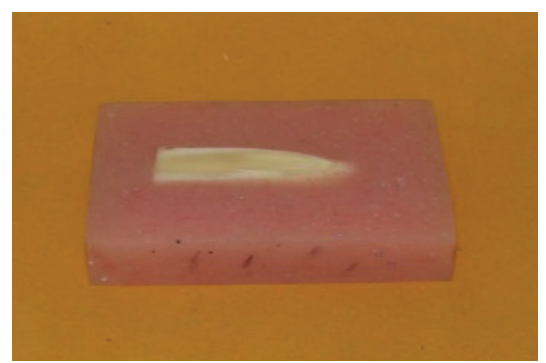

Figura $\mathrm{N}^{\circ} 1$ : espécimen para el estudio. Bloque de acrílico $(4 \mathrm{~cm} \times 3 \mathrm{~cm} \times 0.8 \mathrm{~cm})$ con una superficie de dentina de bobino y todas las superficies pulidas

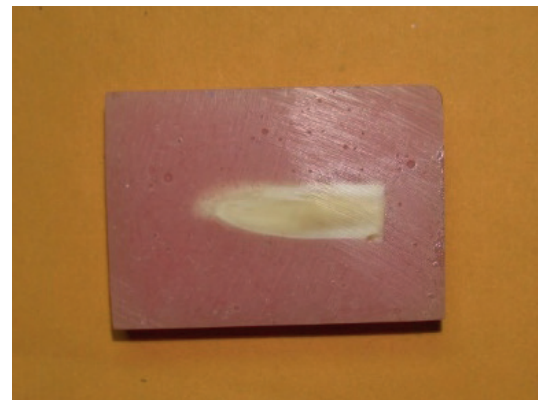

Figura $\mathrm{N}^{\circ}$ 2: espécimen $\mathrm{N}^{\circ} 8$, escogido aleatoriamente con las diferentes soluciones irrigadoras sobre la superficie dentinaria

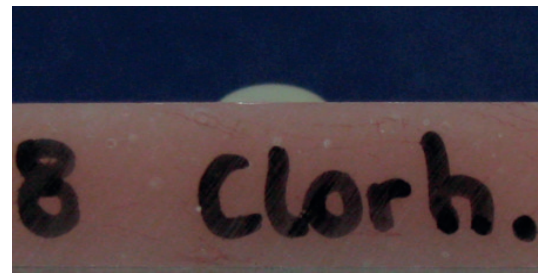

Figura $\mathrm{N}^{\circ} 3$. Clorhexidina al $0.12 \%$

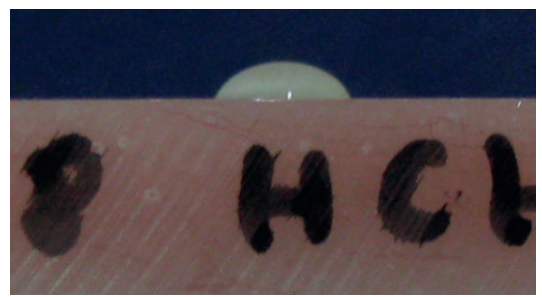

Figura $\mathrm{N}^{\circ} 4$. Hipoclorito de sodio al 5,25\%

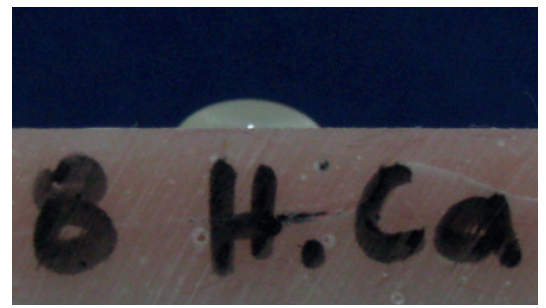

Figura $N^{\circ} 5$. Agua de cal

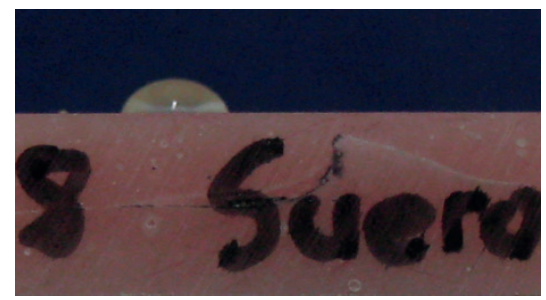

Figura $\mathrm{N}^{\circ}$ 6. Suero fisiológico

\section{Resultados}

El ángulo de contacto de la clorhexidina $0,12 \%$ presenta el menor valor y el mayor valor el hipoclorito de sodio $5,25 \%$. Al comparar los ángulos con la prueba t de Student para muestras relacionadas se halló diferencia significativa entre clorhexidina $0,12 \%$ e hipoclorito de sodio $5,25 \%(\mathrm{p}=0,000)$, también entre el agua de cal e hipoclorito de sodio $5,25 \%(p=0,000)$, pero no hubo diferencia entre cloruro de sodio $9 / 1000 \mathrm{e}$ hipoclorito de sodio 5,25\% ( $\mathrm{p}=0,951)$, Tab.1.

Tabla 1. Ángulo de contacto de soluciones irrigantes del conducto radicular

\begin{tabular}{|c|c|c|c|c|c|c|c|}
\hline & N & Media & Mediana & Moda & $\begin{array}{c}\text { Des. } \\
\text { Tip. }\end{array}$ & Mínimo & Máximo \\
\hline $\begin{array}{c}\text { Clorhexidina } \\
0,12 \%\end{array}$ & 15 & 41,33 & 40,00 & 40 & 3,885 & 36 & 48 \\
\hline $\begin{array}{c}\text { NaOCL } \\
5,25 \%\end{array}$ & 15 & 61,47 & 64,00 & 65 & 9,826 & 29 & 70 \\
\hline $\begin{array}{c}\text { Agua de } \\
\text { cal }\end{array}$ & 15 & 43,07 & 43,00 & 40 & 3,973 & 37 & 50 \\
\hline $\begin{array}{c}\text { Solución } \\
\text { salina }\end{array}$ & 15 & 61,27 & 61,00 & 61 & 3,575 & 55 & 69 \\
\hline
\end{tabular}

\section{Discusión}

El ángulo de contacto es un indicador de la humectabilidad de los liquidos. Los valores experimentales del ángulo de contacto pueden obtenerse mediante medición de la tangente del ángulo entre la gotita de líquido y la superficie sólida del sustrato ${ }^{10}$, la standarización de la superficie permitió comparar la humectabilidad de las soluciones que controlan este sesgo.

Un valor bajo del ángulo de contacto indica que el líquido tiene una alta humectabilidad, mientras que un valor alto indica una baja humectabilidad. Se ha determinado que si el ángulo de contacto es inferior a $90^{\circ}$, se humedece la superficie, si es mayor que $90^{\circ}$, no hay mojado; y un ángulo de contacto igual a cero representa el mojado completo 11. En este estudio, todas las sustancias ensayadas presentaron ángulos de contacto inferiores a $90^{\circ}$.

La humectabilidad es la tendencia de un fluido dado a extenderse o adherirse sobre una superficie sólida. El mojado está correlacionado con el ángulo de contacto cero, mientras que el despliegue está relacionado con la extensión del área proyectada ${ }^{1}$. Esta propiedad es importante para que la solución química penetre los conductos radiculares principales y laterales, así como los túbulos dentinarios, y depende de la tensión superficial ${ }^{8,17}$.

En este estudio, la solución irrigante hipoclorito de sodio al 5,25\% mostró el mayor ángulo de contacto seguido por agua de cal, solución salina y gluconato de clorhexidina al $0.12 \%$ en ese orden. La capacidad de humectabilidad fue significativamente mayor en la clorhexidina al $0.12 \%$ y agua de cal que hipoclorito de sodio al $5,25 \%$, siendo esta última similar al grupo control que el suero fisiológico. Si bien la clorhexidina tiene una buena capacidad humectante y antimicrobiana, no disuelve tejido necrótico remanente, como si lo hace el hipoclorito de sodio ${ }^{13}$.

Las soluciones irrigantes seleccionadas en este estudio fueron probadas en su forma pura, sin la adición de ninguna sustancia, surfactante. Estas sustancias pueden resultar agentes tensioactivos pudiendo reducir la tensión superficial de algunos irrigantes hasta el 50\%, aumentando su humectabilidad en una superficie. Esta es una buena estrategia para aumentar la capacidad del irrigante para penetrar en la dentina del conducto radicular y, en consecuencia, aumentar su acción antimicrobiana. La temperatura y el $\mathrm{pH}$ también afectan la tensión superficial de las soluciones $14,-17$.

Palazzi F, et al. ${ }^{15}$ en el año 2012 hicieron una comparacion de humectabilidad de irrigantes, para lo cual utilizaron hipoclorito de sodio al 5,25\% y tres soluciones a base de hipoclorito de sodio, recientemente introducidas, modificadas para reducir su tensión superficial: Chlor-Xtra, Hypoclean A y Hypoclean $\mathrm{B}$; llegando a la conclusión que las nuevas soluciones de hipoclorito de sodio al 5,25\% modificadas con tensioactivos, Hypoclean A y Hypoclean B, tuvieron valores de humectación significativamente más bajos $(\mathrm{P}<0,01)$ que el Chlor-Xtra y que el hipoclorito de sodio al 5,25\%. Estos nuevos irrigantes tienen el potencial de penetrar más fácilmente en áreas no instrumentadas del sistema de conductos radiculares, así como permitir un intercambio más rápido con solución fresca, lo que permite una mayor eficacia antimicrobiana y capacidad de disolución del tejido pulpar.

No hay duda de que la solución de irrigante ideal debe presentar una buena humectabilidad asociada a una actividad antimicrobiana eficaz ${ }^{13}$.

\section{Conclusiones}

1. La soluciones irrigantes, suero fisiologico y el hipoclorito de sodio al 5,25\% establecieron mayor ángulo de contacto, seguidos por 
agua de cal, y gluconato de clorhexidina al $0.12 \%$ respectivamente

2. La capacidad de humectación del gluconato de clorhexidina al $0,12 \%$ y agua de cal fueron significativamente mejores que el hipoclorito de sodio al 5,25\% y suero fisiológico

3. La capacidad humectante del gluconato de clorhexidina al $0.12 \%$ y el agua de cal fueron muy similares, siendo mejor el primero.

\section{Referencias bibliográficas}

1. Shabahang S, Pouresmail M, Torabinejad M (2003) In vitro antimicrobial efficacy of MTAD and sodium hypochlorite.J Endod 29: 450-452.

2. Machnick TK, Torabinejad M, Munoz CA, Shabahang S (2003) Effect of MTAD on flexural strength and modulus of elasticity of dentin.J Endod 29: 747-750.

3. Shabahang S, Aslanyan J, Torabinejad M (2008) The substitution of chlorhexidine for doxycycline in MTAD: the antibacterial efficacy against a strain of Enterococcus faecalis.J Endod 34: 288-290.

4. Lopez HP, De Faria AR, Alves FRF, Elias CN. Wettability of Irrigants used in Root Canal Treatment. Dentistry 2015; 5: 283. doi: 10.4172/2161-1122.1000283 disponible en: http://www.omicsonline.org/open-access/wettability-of-irrigants-used-in-root-canal- treatment-2161-1122-1000283. php?aid $=40995$

5. Hübscher W, Barbakow F, Peters OA (2003) Root-canal preparation with FlexMaster: canal shapes analysed by micro-computed tomography.IntEndod J 36: 740-747.

6. Peters OA, Peters CI, Schönenberger K, Barbakow F (2003) ProTaper rotary root canal preparation: effects of canal anatomy on final shape analysed by micro CT.IntEndod J 36: 86-92.

7. Zhao D, Shen Y, Peng B, Haapasalo M (2013) Micro-computed tomography evaluation of the preparation of mesiobuccal root canals in maxillary first molars with Hyflex CM, Twisted Files, and $\mathrm{K} 3$ instruments.J Endod 39: 385-388.

8. Giardino L, Ambu E, Becce C, Rimondini L, Morra M (2006) Surface tension comparison of four common root canal irrigants and two new irrigants containing antibiotic.J Endod 32: 1091-1093.

9. Lasala A. Endodoncia. $4^{\mathrm{a}}$ ed. Mexico: Editorial Salvat. 1992.

10. Kumar G, Prabhu KN (2007) Review of non-reactive and reactive wetting of liquids on surfaces.Adv Colloid Interface Sci 133: 61-89.

11. Kontakiotis EG, Tzanetakis GN, Loizides AL (2007) A comparative study of contact angles of four dif- ferent root canal sealers.J Endod 33: 299-302.

12. Steenbecker Gonzales, Oscar y et al. Principios y bases de los biomateriales en Operatoria dental estética adhesiva. Valparaíso: Ed. Universidad de Valparaíso; 2006.

13. Morgana E, Gomes FA, Zaia A.F. In vitro evaluation of the antimicrobial activity of chlorhexidine and sodium hypochlorite.Oral Surg Oral Med Oral Pathol Oral Radiol Endod. 2004;97: 79-84

14. Yılmaz Z, Aktemur S, Buzoglu HD, Gümüsderelioglu M (2011) The effect of temperature and $\mathrm{pH}$ variations on the surface tension of EDTA solutions.J Endod 37: 825-827.

17. Palazzi F, Morra M, Mohammadi Z, Grandini S, Giardino L (2012) Comparison of the surface tension of $5.25 \%$ sodium hypochlorite solution with three new sodium hypochlorite-based endodontic irrigants.IntEndod J 45: 129-135.

16. Wang Z, Shen Y, Ma J, Haapasalo M (2012) The effect of detergents on the antibacterial activity of disinfecting solutions in dentin.J Endod 38: 948-953.

17. Abou-Rass M, Patonai FJ Jr (1982) The effects of decreasing surface tension on the flow of irrigating solutions in narrow root canals.Oral Surg Oral Med Oral Pathol 53: 524-526. 